\title{
Cinética de secagem de folhas de timbó (Serjania marginata Casar)
}

\author{
Elton A. S. Martins ${ }^{1}$, Eduardo Z. Lage ${ }^{2}$, André L. D. Goneli ${ }^{3}$, Cesar P. Hartmann Filho ${ }^{4}$ \& Jéssica G. Lopes ${ }^{5}$
}

\section{Palavras-chave:}

modelo de Midilli

coeficiente de difusão

propriedades termodinâmicas

razão de umidade

\begin{abstract}
R E S U M O
Propôs-se, neste trabalho, avaliar a cinética de secagem de folhas de timbó (Serjania marginata Casar) e ajustar diferentes modelos matemáticos aos valores experimentais de razão de umidade. As folhas de timbó foram colhidas com teor de água inicial de 2,03 \pm 0,10 decimal b.s., sendo submetidas à secagem sob condições controladas de temperatura $\left(40,50,60\right.$ e $\left.70{ }^{\circ} \mathrm{C}\right)$, até o teor de água de 0,08 \pm 0,02 decimal b.s. Aos dados experimentais foram ajustados dez modelos matemáticos utilizados para representação da secagem de produtos agrícolas. Com base nos resultados obtidos o modelo matemático de Midilli é o que melhor representa a cinética de secagem para as folhas de timbó. O aumento da temperatura promove: uma taxa maior de remoção de água nas folhas de timbó durante a secagem; aumento do coeficiente de difusão efetivo sendo que esta relação pode ser descrita pela equação de Arrhenius que apresenta uma energia de ativação para a difusão líquida durante a secagem de $81,39 \mathrm{~kJ} \mathrm{~mol}^{-1}$ para as folhas de timbó; aumento da energia livre de Gibbs, enquanto a entalpia e a entropia decrescem.
\end{abstract}

Key words:

Midilli model

diffusion coefficient

thermodynamic properties

moisture ratio

\section{Drying kinetics of Serjania marginata Casar leaves}

\begin{abstract}
A B S T R A C T
This study aimed to evaluate the kinetics of drying of Serjania marginata leaves, as well as to adjust different mathematical models to the experimental values of the moisture ratio. The Serjania marginata Casar leaves were harvested with initial moisture content of 2.03 $\pm 0,10$ decimal dry basis., and submitted to drying process under controlled conditions of temperature $\left(40,50,60\right.$ and $\left.70{ }^{\circ} \mathrm{C}\right)$, until the moisture content of $0.08 \pm 0,02$. Ten mathematical models were fitted to the experimental data and utilized to predict the drying process of agricultural products. Based on the obtained results, the mathematical model of Midilli best represented the kinetics of drying leaves of Serjania marginata. The increase in temperature promotes: an increased rate of water removal in Serjania marginata leaves during drying; the increase of the effective diffusion coefficient, and this relationship can be described through the Arrhenius equation, which presents an activation energy for the liquid diffusion during drying of $81.39 \mathrm{~kJ} \mathrm{~mol}^{-1}$ for Serjania marginata leaves; an increase in Gibbs free energy, while the enthalpy and entropy decrease.
\end{abstract}




\section{INTRODUÇÃO}

O timbó (Serjania marginata Casar) é uma espécie vegetal de hábito trepador escandente, apresentando grande ocorrência no cerrado brasileiro utilizado tanto para fins ornamentais quanto para o manejo integrado de pragas em locais de recuperação de áreas degradadas devido ao seu potencial inseticida (Guarim Neto \& Santana, 2000). Segundo Bourdy et al. (2004), o timbó possui propriedades medicinais cujo composto de suas folhas é utilizado popularmente para dores de estômago.

$\mathrm{O}$ interesse por produtos com potencial terapêutico, condimentares e aromáticos vem crescendo a cada dia que passa, tanto no Brasil como no mundo (Martinazzo et al., 2007; Tabaldi et al., 2012). Devido a esta demanda acaba se tornando essencial, além das práticas agrícolas sustentáveis e mais eficientes, o desenvolvimento de técnicas eficientes de secagem e armazenamento para que a biomassa produzida pela planta e suas propriedades químicas possam ser aproveitadas de forma integral e efetiva (Tabaldi et al., 2012).

A secagem de plantas medicinais é uma operação que tem a finalidade de preparar as plantas para um armazenamento seguro, garantindo-lhes a permanência de suas propriedades medicinais, visando atender às necessidades das indústrias farmacêuticas de fitoterápicos, os quais não têm meios de utilizar plantas frescas na quantidade exigida para a produção industrial (Lorenzi \& Matos, 2008).

De acordo com Lorenzi \& Matos (2008), a secagem de plantas medicinais deve ocorrer à sombra, em área coberta, limpa e ventilada, distribuindo as folhas de modo a formar camadas finas que, por sua vez, devem ser revolvidas periodicamente visando uniformizar sua secagem que, em geral, demora de 3 a 5 dias. A secagem é um processo que pode demorar desde algumas horas até vários dias dependendo de alguns fatores como: método de secagem, temperatura, umidade relativa e velocidade do ar de secagem (Dalpasquale \& Sperandio, 2010). Sendo os principais fatores que afetam o processo, a temperatura e a velocidade do ar visto que, quanto maior forem esses fatores menor será o tempo de secagem do produto, tal como também a possibilidade de perda de qualidade devido ao estresse hídrico gerado pela elevada taxa de secagem (Carlesso et al., 2005).

De acordo com Martinazzo (2006), o processo de secagem em camada delgada ou fina é definido como aquele com espessura de apenas uma unidade do produto e considera que uma camada espessa seja constituída de uma sucessão de camadas delgadas superpostas; desta forma, a equação de secagem em camada delgada, combinada com as equações representativas de outras propriedades físicas específicas do produto em estudo, forma um conjunto de relações matemáticas que auxiliam nos cálculos e no entendimento dos processos de secagem em camada espessa.

Em estudos sobre a secagem de produtos agrícolas em camada delgada são usados três tipos de modelo sendo eles: o modelo teórico, que considera apenas a resistência interna à transferência de calor e água entre o produto e o ar quente, os modelos semiteóricos e os empíricos, que ponderam somente a resistência externa a temperatura e umidade relativa do ar de secagem (Midilli et al., 2002; Panchariya et al., 2002).

Conhecer as propriedades termodinâmicas nos processos de secagem de produtos agrícolas é uma fonte de informação importante para projetar equipamentos de secagem, calcular a energia requerida neste processo, estudar as propriedades da água adsorvida e avaliar a microestrutura dos alimentos e o estudo dos fenômenos físicos que ocorrem na superfície dos produtos agrícolas (Corrêa et al., 2010).

Desta forma, o presente trabalho foi desenvolvido com o objetivo de ajustar e modelar, estatisticamente, os modelos matemáticos e suas características termodinâmicas durante a cinética de secagem de folhas de timbó.

\section{Material e Métodos}

O experimento foi conduzido no laboratório de Propriedades Físicas de Produtos Agrícolas, da Faculdade de Ciências Agrárias - FCA, pertencente à Universidade Federal da Grande Dourados, no município de Dourados, MS. Foram utilizadas folhas da parte aérea de timbó, colhidas no Horto de Plantas Medicinais - HPM da FCA.

As folhas foram colhidas nas primeiras horas da manhã após não haver mais orvalho sobre suas superfícies. $\mathrm{Na}$ época de coleta das folhas não foi realizada a irrigação nem foram coletadas folhas após precipitações pluviométricas visando evitar variações no seu teor de água inicial. A coleta foi realizada de modo aleatório; posteriormente foram selecionadas folhas com ausência de partes danificadas e homogeneizadas com a finalidade de evitar qualquer tipo de interferência nos resultados.

O teor de água inicial e de equilíbrio das amostras foi determinado logo após a coleta e no final do processo de secagem, respectivamente, utilizando-se o método gravimétrico recomendado pela ASABE (2010), para forrageiras e plantas similares, em estufa com circulação forçada de ar a $103 \pm 1{ }^{\circ} \mathrm{C}$ durante $24 \mathrm{~h}$.

O produto foi submetido a quatro temperaturas controladas de ar de secagem sendo: 40, 50, 60 e $70{ }^{\circ} \mathrm{C}$, com respectivas umidades relativas do ar de secagem de 19,$2 ; 16,4 ; 9,3$ e 4,8\%, em uma estufa com circulação forçada de ar até que o produto atingisse o teor de água de equilíbrio. A temperatura do ar de secagem foi monitorada por um termômetro ordinário de mercúrio e a umidade relativa no interior da estufa foi calculada utilizando-se o software Grapsi versão 8.1.1, com base em dados das condições do ambiente externo o qual foi monitorado periodicamente durante o processo de secagem, utilizando-se três termo-higrômetros.

O teor de água inicial das folhas de timbó submetidas aos ensaios de secagem foi de 2,03 $\pm 0,10$ decimal b.s. Para fins de modelagem matemática, uma vez que ao final do processo de secagem ocorre grande variação de tempo para pouca variação de razão de umidade, prejudicando o ajuste matemático aos dados observados, considerou-se o fim da secagem quando o teor de água do produto esteve em 0,08 \pm 0,02 decimal b.s..

Foram colocadas, no interior da estufa, três bandejas removíveis com fundo telado para permitir a passagem do ar através da camada de produto em que cada bandeja, com aproximadamente $35 \mathrm{~g}$ de produto, se constituiu em uma repetição totalizando, assim, três repetições para cada temperatura de ar de secagem avaliada. Durante o processo de secagem as bandejas com as amostras foram pesadas periodicamente através de uma balança analítica digital com resolução de 0,01 g. O tempo inicial entre as pesagens foi de 10 em 10 min nas duas primeiras horas de experimento; posteriormente, o tempo entre as pesagens foi controlado por meio da diferença de massas (conhecendo-se a massa e o teor de água inicial das folhas), de forma que não permitisse diferenças 
grandes de teor de água entre as leituras. Para o ensaio na temperatura de $70{ }^{\circ} \mathrm{C}$ o tempo entre as leituras de massa foi de 7 em 7 min em virtude do tempo de secagem ser relativamente menor com relação às demais temperaturas obtendo-se, então, uma quantidade suficiente de dados para realizar o ajuste dos modelos matemáticos por regressão não linear.

Para calcular a razão de umidade (RU), nas diferentes temperaturas de secagem, utilizou-se a Eq. 1.

$$
R U=\frac{U-U_{e}}{U_{i}-U_{e}}
$$

sendo:

RU - razão de umidade do produto, adimensional

U - teor de água do produto em determinado tempo, decimal (b.s.)

$\mathrm{U}_{\mathrm{e}} \quad$ - teor de água de equilíbrio do produto, decimal (b.s.)

$\mathrm{U}_{\mathrm{i}} \quad$ - teor de água inicial do produto, decimal (b.s.)

Aos dados de razão de umidade, obtidos experimentalmente durante a secagem de folhas de timbó, foram ajustados 10 modelos matemáticos para predizer o fenômeno da secagem de produtos agrícolas (Tabela 1).

Tabela 1. Modelos matemáticos ajustados às curvas de secagem de folhas de timbó (Midilli et al., 2002; Corrêa et al., 2007; Martinazzo et al., 2007; Goneli et al., 2014b)

\begin{tabular}{lll}
\hline \multicolumn{1}{c}{ Designação do modelo } & \multicolumn{1}{c}{ Modelo } \\
Aproximação da difusão & $\mathrm{RU}=\mathrm{a} \exp (-\mathrm{k} \theta)+(1-\mathrm{a}) \exp (-\mathrm{k} \mathrm{b} \theta)$ & $(2)$ \\
Dois termos & $\mathrm{RU}=\mathrm{a} \exp \left(-\mathrm{k}_{0} \theta\right)+\mathrm{b} \exp \left(-\mathrm{k}_{1} \theta\right)$ & $(3)$ \\
Exponencial de dois termos & $\mathrm{RU}=\mathrm{a} \exp (-\mathrm{k} \theta)+(1-\mathrm{a}) \exp (-\mathrm{k} \mathrm{a} \theta)$ & $(4)$ \\
Henderson e Pabis & $\mathrm{RU}=\mathrm{a} \exp (-\mathrm{k} \theta)$ & $(5)$ \\
Logarítmico & $\mathrm{RU}=\mathrm{a} \exp (-\mathrm{k} \theta)+\mathrm{c}$ & $(7)$ \\
Midilli & $\mathrm{RU}=\mathrm{a} \exp \left(-\mathrm{k} \theta^{\mathrm{n}}\right)+\mathrm{b} \theta$ & $(8)$ \\
Newton & $\mathrm{RU}=\exp (-\mathrm{k} \theta)$ & $(9)$ \\
Page & $\mathrm{RU}=\exp \left(-\mathrm{k} \theta^{\mathrm{n}}\right)$ & $(10)$ \\
Thompson & $\mathrm{RU}=\exp \left\{\left[-\mathrm{a}-\left(\mathrm{a}^{2}+4 \mathrm{~b} \theta\right)^{0,5}\right] / 2 \mathrm{~b}\right\}$ & $(11)$ \\
Wang e Singh & $\mathrm{RU}=1+\mathrm{a} \theta+\mathrm{b} \theta^{2}$ & \\
\hline$\theta$ - tempo de secagem, $\mathrm{h} ; \mathrm{k}, \mathrm{k}_{\mathrm{o}}, \mathrm{k}_{1}-\operatorname{constantes~de~secagem,~} \mathrm{h}^{-1} ; \mathrm{a}, \mathrm{b}, \mathrm{c}, \mathrm{n}$ - coeficientes dos \\
modelos
\end{tabular}

Os coeficientes de difusão efetivo das folhas de timbó para as diferentes temperaturas de secagem $\left(40,50,60\right.$ e $\left.70{ }^{\circ} \mathrm{C}\right)$ foram calculados utilizando-se a Eq. 12 baseada na teoria da difusão líquida. Esta equação é a solução analítica para a segunda lei de Fick considerando-se a forma geométrica do produto como aproximada de uma placa plana e com aproximação de oito termos.

$$
\begin{aligned}
R U & =\frac{U-U_{e}}{U_{i}-U_{e}}= \\
& =\frac{8}{\pi^{2}} \sum_{n=0}^{\infty} \frac{1}{(2 n+1)^{2}} \exp \left[-(2 n+1)^{2} \pi^{2} D_{i}\left(\frac{X}{4 L}\right)^{2}\right]
\end{aligned}
$$

sendo:

$$
\begin{array}{ll}
\mathrm{D}_{\mathrm{i}} & \text { - coeficiente de difusão efetivo, } \mathrm{m}^{2} \mathrm{~s}^{-1} \\
\mathrm{X} & \text { - tempo de secagem, } \mathrm{s} \\
\mathrm{L} & \text { - espessura do produto, } \mathrm{m} \\
\mathrm{n} & \text { - número de termos do modelo }
\end{array}
$$

A espessura das folhas de timbó foi mensurada utilizandose um paquímetro digital com resolução de $0,01 \mathrm{~mm}$. Foram realizadas medições em 50 folhas sendo que em cada folha foram realizadas três medições em diferentes locais; após isto foi calculada a média dos valores mensurados sendo esta média a espessura das folhas, que foi de $0,5922 \mathrm{~mm}$.

Para avaliar a influência da temperatura no coeficiente de difusão efetivo foi utilizada a equação de Arrhenius, descrita na Eq. 13:

$$
\mathrm{D}_{\mathrm{i}}=\mathrm{D}_{0} \exp \left(\frac{\mathrm{E}_{\mathrm{a}}}{\mathrm{RT}_{\mathrm{a}}}\right)
$$

sendo:

$\mathrm{D}_{\mathrm{o}}$ - fator pré-exponencial

$\mathrm{T}_{\mathrm{a}}$ - temperatura absoluta, $\mathrm{K}$

$\mathrm{R}^{\mathrm{a}}$ - constante universal dos gases, $8,314 \mathrm{~kJ} \mathrm{kmol}^{-1} \mathrm{~K}^{-1}$

$\mathrm{E}_{\mathrm{a}} \quad$ - energia de ativação, $\mathrm{kJ} \mathrm{mol}^{-1}$

As propriedades termodinâmicas entalpia específica, entropia específica e energia livre de Gibbs, relacionadas ao processo de secagem das folhas de timbó, foram determinadas através do método descrito por Jideani \& Mpotokwana (2009) de acordo com as Eqs. 14, 15 e 16 descritas abaixo.

$$
\begin{gathered}
\Delta \mathrm{h}=\mathrm{E}_{\mathrm{a}}-\mathrm{RT}_{\mathrm{a}} \\
\Delta \mathrm{s}=\mathrm{R}\left(\ln \mathrm{D}_{0}-\ln \frac{\mathrm{k}_{\mathrm{B}}}{\mathrm{h}_{\mathrm{p}}}-\ln \mathrm{T}_{\mathrm{a}}\right) \\
\Delta \mathrm{G}=\Delta \mathrm{h}-\mathrm{T}_{\mathrm{a}} \Delta \mathrm{s}
\end{gathered}
$$

sendo:

$\Delta \mathrm{h}$ - entalpia específica, $\mathrm{J} \mathrm{mol}^{-1}$

$\Delta s \quad$ - entropia específica, $\mathrm{J} \mathrm{mol}^{-1} \mathrm{~K}^{-1}$

$\Delta \mathrm{G}$ - energia livre de Gibbs, $\mathrm{J} \mathrm{mol}^{-1}$

$\mathrm{k}_{\mathrm{B}} \quad$ - constante de Boltzmann, 1,38 x 10 $0^{-23} \mathrm{~J} \mathrm{~K}^{-1}$

$\mathrm{h}_{\mathrm{p}} \quad$ - constante de Planck, 6,626 x 10-34 $\mathrm{J} \mathrm{s}^{-1}$

Os dados experimentais de cinética de secagem das folhas de timbó foram submetidos à análise de regressão não linear, pelo método de Gauss-Newton, e seleção do modelo matemático adequado para expressar a relação entre as variáveis estudadas. Para o ajuste dos modelos matemáticos aos dados experimentais utilizou-se o programa computacional Statistica $8.0^{\circledR}$.

Para a análise do grau de ajuste de cada modelo foram consideradas as magnitudes do coeficiente de determinação ajustado, do erro médio relativo, do desvio padrão da estimativa e o comportamento da distribuição dos resíduos.

Os valores do erro médio relativo $(\mathrm{P})$ e do desvio padrão da estimativa (SE) foram calculados conforme descrito pelas Eqs. 17 e 18.

$$
\begin{aligned}
& P=\frac{100}{N} \sum_{i=1}^{N}\left(\frac{|Y-\hat{Y}|}{Y}\right) \\
& S E=\sqrt{\frac{\sum_{i=1}^{N}(Y-\hat{Y})^{2}}{G L R}}
\end{aligned}
$$


sendo:

$\mathrm{N}$ - número de observações experimentais

Y - valor estimado pelo modelo

Y - valor experimental

GLR - graus de liberdade do modelo.

\section{Resultados E Discussão}

Em todas as temperaturas utilizadas na secagem de folhas de timbó os modelos ajustados aos dados experimentais apresentaram valores de coeficiente de determinação acima de 0,95 (Tabela 2) o que, segundo Kashaninejad et al. (2007) indica uma representação satisfatória desses modelos para o processo de secagem estudado. Madamba et al. (1996) afirmam que o uso do valor do coeficiente de determinação como único critério não é um bom parâmetro para a seleção de modelos matemáticos não lineares para representar o processo de secagem tornando-se necessária a análise conjunta de outros parâmetros estatísticos.

Dentre os modelos ajustados o único modelo que apresentou valores de erro médio relativo $(\mathrm{P})$ menor que $10 \%$, no processo de secagem de folhas de timbó para todas as temperaturas estudadas, foi o modelo de Midilli (7) (Tabela 2). Segundo Kashaninejad et al. (2007) os valores do erro médio relativo (P) indicam o desvio dos dados observados a partir da curva estimada pelo modelo. Mohapatra \& Rao (2005) ressaltam que modelos que apresentam valores de erro médio relativo superior a $10 \%$ são inadequados para representar o processo de secagem.

Os modelos Logarítmico (6) e de Midilli (7) apresentaram os menores valores de desvio padrão da estimativa (Tabela 2). De acordo com Siqueira et al. (2012) quanto menores os valores de SE melhor o ajuste do modelo aos dados observados. Os modelos Logarítmico (6) e de Midilli (7) também apresentam distribuição aleatória dos resíduos para todas as temperaturas do ar de secagem (Tabela 2). Segundo Goneli (2008) um modelo é considerado aceitável se apresentar a distribuição dos resíduos aleatória, ou seja, se os valores dos resíduos se encontrarem próximos à faixa horizontal em torno de zero e, caso o modelo apresente distribuição dos resíduos de forma tendenciosa, o mesmo não é adequado para representar o fenômeno em questão.

Verifica-se, com base em todos os parâmetros estatísticos analisados, que os modelos Logarítmico (6) e Midilli (7) apresentaram ajuste satisfatório aos dados experimentais para as temperaturas de 40 e $50{ }^{\circ} \mathrm{C}$ porém apenas o modelo de Midilli (7) apresentou bom ajuste para as temperaturas de 60 e $70{ }^{\circ} \mathrm{C}$; recomenda-se, então, o modelo de Midilli para predizer o processo de secagem em camada delgada de folhas de timbó haja vista que o mesmo apresentou bom ajuste para todas as condições de secagem estudadas neste trabalho.

Radünz et al. (2011) ajustaram, avaliando a cinética de secagem de carqueja, 12 modelos matemáticos e concluíram, usando como parâmetros para a seleção do modelo o coeficiente de determinação, o erro médio relativo e o desvio padrão da estimativa, que o modelo de Midilli foi o que melhor se ajustou enquanto os demais modelos apresentaram valores de erro médio relativo superiores a $10 \%$ em pelo menos uma das temperaturas analisadas. O modelo de Midilli também foi o que melhor se ajustou à secagem de folhas de aroeira estudada por Goneli et al. (2014b), na secagem de folhas de fruta-de-lobo estudada por Prates et al. (2012) e na secagem de capim limão estudado por Martinazzo et al. (2007). Segundo Goneli et al. (2014b) o melhor ajuste do modelo de Midilli aos dados experimentais de secagem de plantas medicinais está ligado, provavelmente, à rápida perda de água nos estádios iniciais do processo neste tipo de produto gerando uma curva de secagem mais acintosa e mais bem caracterizada matematicamente, por este modelo.

$\mathrm{O}$ aumento da temperatura do ar de secagem reduz diretamente o tempo necessário para a remoção de água do produto (Figura 1) diminuindo, assim, o tempo de secagem. Este fenômeno foi observado por diversos pesquisadores em vários produtos agrícolas (Martinazzo et al., 2007; Radünz et al., 2011; Prates et al., 2012; Siqueira et al., 2012; Goneli et al., 2014b).

Tabela 2. Parâmetros estatísticos (SE - desvio padrão da estimativa, P - erro médio relativo e $\mathrm{R}^{2}$ - coeficiente de determinação) e distribuição dos resíduos (TD = tendenciosa; $\mathrm{AL}$ = aleatória) para os dez modelos ajustados às curvas de secagem de folhas de timbó

\begin{tabular}{|c|c|c|c|c|c|c|c|c|}
\hline Modelos & $\begin{array}{c}\text { SE } \\
\text { (decimal) }\end{array}$ & $\begin{array}{l}P \\
(\%)\end{array}$ & $\begin{array}{c}\mathrm{R}^{2} \\
\text { (decimal) }\end{array}$ & $\begin{array}{l}\text { Distribuição } \\
\text { dos resíduos }\end{array}$ & $\begin{array}{c}\text { SE } \\
\text { (decimal) }\end{array}$ & $\begin{array}{l}P \\
(\%)\end{array}$ & $\begin{array}{c}\mathrm{R}^{2} \\
\text { (decimal) }\end{array}$ & $\begin{array}{l}\text { Distribuição } \\
\text { dos resíduos }\end{array}$ \\
\hline & \multicolumn{4}{|c|}{$40^{\circ} \mathrm{C}$} & \multicolumn{4}{|c|}{$50^{\circ} \mathrm{C}$} \\
\hline (2) & 0,0288 & 18,3366 & 0,9920 & TD & 0,0156 & 15,4581 & 0,9981 & $\mathrm{AL}$ \\
\hline (3) & 0,0244 & 15,8718 & 0,9944 & TD & 0,0165 & 15,6466 & 0,9982 & $\mathrm{AL}$ \\
\hline (4) & 0,0323 & 20,5100 & 0,9913 & TD & 0,0152 & 17,3491 & 0,9980 & $\mathrm{AL}$ \\
\hline (5) & 0,0416 & 30,7243 & 0,9828 & TD & 0,0299 & 35,6977 & 0,9925 & TD \\
\hline (6) & 0,0125 & 8,5036 & 0,9985 & $A L$ & 0,0159 & 9,5847 & 0,9981 & $\mathrm{AL}$ \\
\hline (7) & 0,0131 & 7,1517 & 0,9984 & $A L$ & 0,0126 & 5,9398 & 0,9989 & $\mathrm{AL}$ \\
\hline (8) & 0,0424 & 32,7599 & 0,9816 & TD & 0,0327 & 40,3597 & 0,9900 & TD \\
\hline (9) & 0,0308 & 18,5508 & 0,9906 & TD & 0,0141 & 15,4276 & 0,9983 & $\mathrm{AL}$ \\
\hline (10) & 0,0430 & 32,7621 & 0,9816 & TD & 0,0343 & 40,3669 & 0,9900 & TD \\
\hline \multirow[t]{2}{*}{ (11) } & 0,0178 & 3,1090 & 0,9969 & TD & 0,0228 & 18,9601 & 0,9956 & TD \\
\hline & \multicolumn{4}{|c|}{$60^{\circ} \mathrm{C}$} & \multicolumn{4}{|c|}{$70^{\circ} \mathrm{C}$} \\
\hline (2) & 0,0330 & 30,5777 & 0,9946 & TD & 0,0339 & 26,9164 & 0,9952 & TD \\
\hline (3) & 0,0888 & 68,8266 & 0,9705 & TD & 0,1064 & 65,9551 & 0,9686 & TD \\
\hline (4) & 0,0327 & 34,7260 & 0,9933 & TD & 0,0329 & 30,9251 & 0,9940 & TD \\
\hline (5) & 0,0688 & 68,8266 & 0,9705 & TD & 0,0747 & 65,4678 & 0,9691 & TD \\
\hline (6) & 0,0224 & 19,2416 & 0,9975 & $\mathrm{AL}$ & 0,0292 & 21,6274 & 0,9964 & $\mathrm{AL}$ \\
\hline (7) & 0,0141 & 9,0192 & 0,9993 & $A L$ & 0,0175 & 8,3537 & 0,9991 & $\mathrm{AL}$ \\
\hline (8) & 0,0674 & 73,9101 & 0,9660 & TD & 0,0707 & 69,4878 & 0,9654 & TD \\
\hline (9) & 0,0243 & 22,4068 & 0,9963 & TD & 0,0227 & 17,8921 & 0,9971 & $\mathrm{AL}$ \\
\hline (10) & 0,0738 & 73,9130 & 0,9660 & TD & 0,0790 & 69,4925 & 0,9654 & TD \\
\hline (11) & 0,0146 & 12,3766 & 0,9987 & $\mathrm{AL}$ & 0,0194 & 14,5407 & 0,9979 & $\mathrm{AL}$ \\
\hline
\end{tabular}




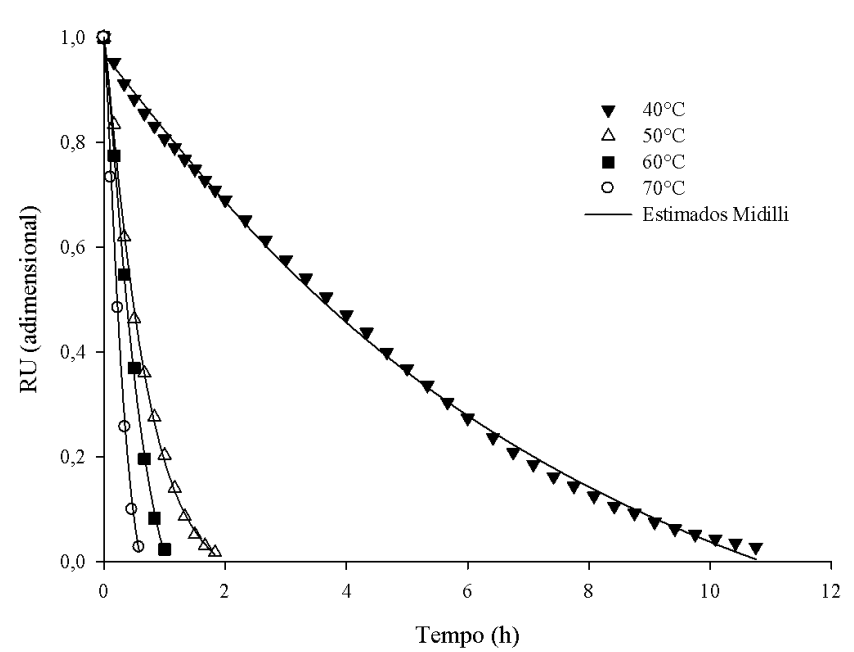

Figura 1. Valores de razão de umidade (RU) experimentais e estimados, pelo modelo de Midilli, para o processo de secagem das folhas de timbó

Para que as folhas de timbó atingissem o teor de água de 0,08 $\pm 0,02$ decimal b.s. foram necessários 10,$8 ; 1,8 ; 1,0$ e $0,6 \mathrm{~h}$ para as temperaturas de $40,50,60$ e $70^{\circ} \mathrm{C}$ (Figura 1), respectivamente. Além disto, também é possível observar a proximidade entre valores observados e os estimados pelo modelo de Midilli (Figura 1) reforçando a aplicabilidade deste modelo na predição dos dados de secagem de folhas de timbó para o intervalo de dados estudado.

Os parâmetros "k" e "n" do modelo de Midilli apresentaram variação da magnitude de seus valores em função da temperatura do ar de secagem (Tabela 3). Os parâmetros " $k$ " e " $n$ " tiveram um incremento em seus valores conforme se aumentou a temperatura do ar de secagem; já para os parâmetros "a" e "b" não se observou tendência alguma definida. O parâmetro " $k$ " pode ser usado como aproximação para caracterizar o efeito da temperatura e está relacionado com a difusividade efetiva no processo de secagem no período decrescente, sendo que a difusão líquida controla o processo (Babalis \& Belessiotis, 2004). Conforme a magnitude do parâmetro " $k$ " seja elevada em razão do aumento da temperatura do ar de secagem, a difusividade efetiva também é elevada (Tabela 3), ou seja, quanto maior a magnitude do parâmetro " $k$ " maior a difusividade efetiva no processo de secagem.

Os parâmetros "k" e "n", do modelo de Midilli, na secagem de folhas de timbó podem ser descritos por equações lineares em função da temperatura do ar de secagem. Utilizando essas equações lineares, juntamente com a média dos valores dos parâmetros "a" e "b" e as substituindo nas Eqs. 1 e 7, obtém-se a Eq. 19 para estimar o teor de água das folhas de timbó em função do tempo $(\theta)$, em horas, e da temperatura do ar de secagem (T), em ${ }^{\circ} \mathrm{C}$, para a faixa de temperatura estudada.

Tabela 3. Parâmetros do modelo de Midilli e coeficiente de difusão efetivo para as diferentes temperaturas de secagem das folhas de timbó

\begin{tabular}{|c|c|c|c|c|c|}
\hline \multirow{2}{*}{$\begin{array}{c}\text { Temperatura } \\
\left({ }^{\circ} \mathrm{C}\right)\end{array}$} & \multicolumn{4}{|c|}{ Parâmetros do modelo de Midilli } & \multirow{2}{*}{$\begin{array}{c}D \times 10^{-11} \\
\left(\mathrm{~m}^{2} \mathrm{~s}^{-1}\right)\end{array}$} \\
\hline & $A$ & k & $\mathrm{n}$ & b & \\
\hline 40 & 0,9660 & 0,1435 & 1,0724 & 0,0139 & 0,6630 \\
\hline 50 & 1,0061 & 1,5708 & 1,1239 & 0,0168 & 5,1229 \\
\hline 60 & 0,9979 & 2,1833 & 1,2687 & 0,0980 & 7,0289 \\
\hline 70 & 0,9974 & 4,8315 & 1,3355 & 0,1300 & 12,0712 \\
\hline
\end{tabular}

$$
\begin{aligned}
\mathrm{U} & =\mathrm{U}_{\mathrm{e}}+\left(\mathrm{U}_{\mathrm{i}}-\mathrm{U}_{\mathrm{e}}\right)\{0,9918 \exp [-(0,1468 \mathrm{~T}- \\
& \left.\left.-5,8898) \theta^{0,0093 \mathrm{~T}+0,6864}\right]-0,0647 \theta\right\}
\end{aligned}
$$

Os valores do coeficiente de difusão efetivo aumentam com o incremento da temperatura do ar de secagem (Tabela 3), comportamento este também observado por vários pesquisadores estudando plantas medicinais (Martinazzo et al., 2007; Prates et al., 2012; Goneli et al., 2014b). Goneli (2008), explica que, ocorrendo um aumento da temperatura aumentase também o nível de vibração das moléculas de água e se diminui sua viscosidade a qual é uma medida da resistência do fluido ao escoamento; as variações desta propriedade implicam em alterações na difusão da água nos capilares dos produtos agrícolas que, juntamente com a vibração mais intensa das moléculas de água, contribuem para uma difusão mais rápida.

A variação dos valores do coeficiente de difusão efetivo para a secagem de folhas de timbó numa faixa de temperatura entre 40 e $70^{\circ} \mathrm{C}$ ficou entre $0,6630 \times 10^{-11}$ e $12,0712 \times 10^{-11} \mathrm{~m}^{2} \mathrm{~s}^{-1}$, respectivamente. De acordo com Madamba et al. (1996) os valores do coeficiente de difusão efetiva para a secagem de produtos agrícolas se apresentam na ordem de $10^{-9}$ a $10^{-11} \mathrm{~m}^{2} \mathrm{~s}^{-1}$, corroborando com o presente trabalho. Goneli et al. (2014b) observaram, estudando a secagem de folhas de aroeira e Goneli et al. (2014a) a secagem de folhas de erva baleeira, que para as folhas de aroeira a variação do coeficiente de difusão efetivo ficou entre $0,1476 \times 10^{-11} \mathrm{e} 1,5811 \times 10^{-11} \mathrm{~m}^{2} \mathrm{~s}^{-1}$; já para as folhas de erva baleeira se manteve entre $1,1294 \times 10^{-11}$ e $9,4933 \times 10^{-11}$ $\mathrm{m}^{2} \mathrm{~s}^{-1}$, ambas para uma faixa de temperatura de 40 a $70{ }^{\circ} \mathrm{C}$. A variação do coeficiente de difusão efetivo das folhas de erva baleeira e das folhas de timbó, foi semelhante quando comparada com a dos valores apresentados pelas folhas de aroeira sendo, em média, a área foliar das folhas de timbó e a erva baleeira, semelhantes e superiores à área foliar das folhas de aroeira. As folhas de erva baleeira e timbó apresentaram espessura média de 0,6623 e $0,5922 \mathrm{~mm}$, respectivamente, enquanto as folhas de aroeira apresentaram espessura média de $0,3631 \mathrm{~mm}$. Além das características físicas diferentes entre os produtos, diversos outros fatores também podem influenciar na obtenção do coeficiente de difusão efetivo, como temperatura do ar de secagem, variedade e composição dos materiais (Rizvi, 1995).

A dependência do coeficiente de difusão efetivo com relação à temperatura do ar de secagem tem sido descrita satisfatoriamente pela equação de Arrhenius (Martinazzo et al., 2007; Goneli et al., 2014b). A inclinação da curva de Arrhenius (Figura 2), gerada a partir dos valores de $\ln (\mathrm{D})$ em função do inverso da temperatura absoluta $\left(1 / \mathrm{K}^{-1}\right)$ durante a secagem das folhas de timbó, fornece a relação $\mathrm{Ea} / \mathrm{R}$ enquanto sua interseção com o eixo das ordenadas indica o valor de $\mathrm{D}$.

A Eq. 20 apresenta o coeficiente da equação de Arrhenius ajustada para os coeficientes de difusão efetiva das folhas de timbó, calculado de acordo com a Eq. 13.

$$
\mathrm{D}_{\mathrm{i}}=0,3851 \exp \left(\frac{81389,7572}{\mathrm{RT}_{\mathrm{a}}}\right)
$$

A energia de ativação para a difusão da água durante a secagem das folhas de timbó foi de $81,39 \mathrm{~kJ} \mathrm{~mol}^{-1}$ (Eq. 20). Este valor se apresenta mais elevado do que o calculado por Goneli 


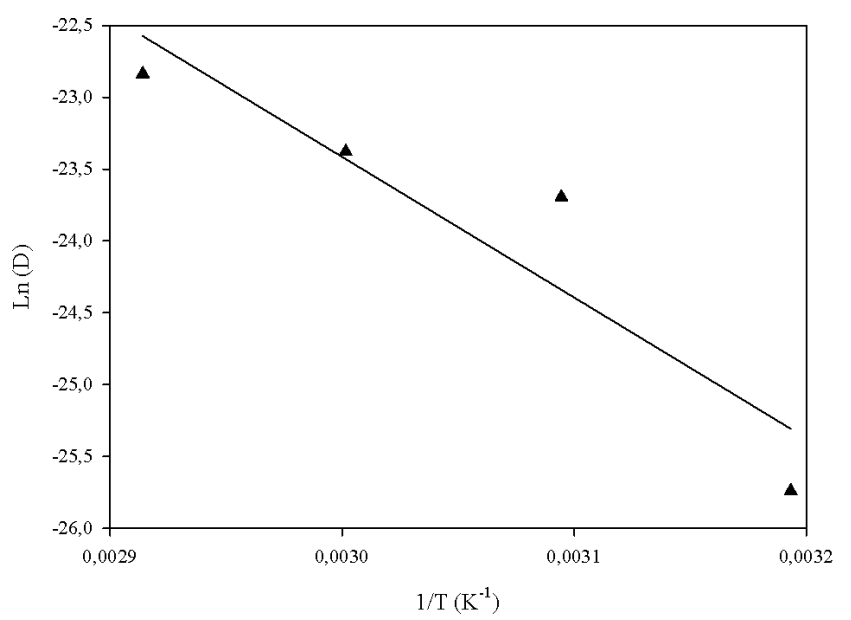

Figura 2. Representação de Arrhenius para o coeficiente de difusão efetivo, em função da temperatura do ar, durante a secagem das folhas de timbó

et al. (2014b) que obtiveram, avaliando a secagem de folhas de aroeira, uma energia de ativação de $74,96 \mathrm{~kJ} \mathrm{~mol}^{-1}$ e por Martinazzo et al. (2007) que calcularam, na secagem de folhas de capim-limão, a energia de ativação em $63,47 \mathrm{~kJ} \mathrm{~mol}^{-1}$. O valor de energia de ativação mais elevado para as folhas de timbó evidenciou a necessidade de maior energia para desencadear o processo difusão de líquido quando comparado com as folhas de aroeira e capim limão. Os diferentes valores de energia de ativação para diferentes produtos agrícolas podem ser atribuídos a características físicas e biológicas dos produtos. Corrêa et al. (2007) conceituam, termodinamicamente, que a energia de ativação é a facilidade com que as moléculas de água superam a barreira de energia durante a migração no interior do produto sendo que quanto menor a energia de ativação maior será a difusividade de água no produto no processo de secagem.

Segundo Zogzas et al. (1996) os valores de energia de ativação para produtos agrícolas variam de 12,7 a $110 \mathrm{~kJ} \mathrm{~mol}^{-1}$, logo o valor de energia de ativação encontrado no presente trabalho está situado nesta faixa de valores propostos por esses autores.

Com relação às propriedades termodinâmicas (entalpia específica, entropia específica e energia livre de Gibbs) observase que os valores de entalpia diminuem com o incremento dos valores de temperatura (Tabela 4), indicando que é necessária uma quantidade menor de energia para que a secagem ocorra em temperaturas mais elevadas.

Analisando a variação dos valores de entropia (Tabela 4) observa-se comportamento semelhante ao da entalpia, ou seja, a entropia reduziu com o aumento da temperatura do ar de secagem. Este comportamento era um fenômeno esperado pois a diminuição da temperatura acarreta em menor excitação das moléculas de água resultando em um aumento da ordem do sistema água-produto (Corrêa et al., 2010). Segundo Goneli

Tabela 4. Propriedades termodinâmicas do processo de secagem das folhas de timbó: entalpia específica $(\Delta h)$, entropia específica $(\Delta s)$ e energia livre de Gibbs $(\Delta \mathrm{G})$

\begin{tabular}{|c|c|c|c|}
\hline $\begin{array}{c}\text { Temperatura } \\
\left({ }^{\circ} \mathrm{C}\right)\end{array}$ & $\begin{array}{c}\Delta \mathrm{h} \\
\left(\mathrm{kJ} \mathrm{mol} \mathbf{~}^{-1}\right)\end{array}$ & $\begin{array}{c}\Delta S \\
\left(\mathrm{KJ} \mathrm{mol}^{-1} \mathrm{~K}^{-1}\right)\end{array}$ & $\begin{array}{c}\Delta G \\
\left(\mathrm{~kJ} \mathrm{~mol}^{-1}\right)\end{array}$ \\
\hline 40 & 78,7861 & $-0,1958$ & 140,1081 \\
\hline 50 & 78,7030 & $-0,1961$ & 142,0676 \\
\hline 60 & 78,6199 & $-0,1963$ & 144,0297 \\
\hline 70 & 78,5367 & $-0,1966$ & 145,9942 \\
\hline
\end{tabular}

et al. (2010), a entropia é uma grandeza termodinâmica ligada ao grau de desordem cujos valores se elevam durante o processo natural em um sistema isolado. Os valores negativos de entropia podem estar atribuídos à existência de adsorção química e/ou modificações estruturais do adsorvente (Moreira et al., 2008).

Diferentemente dos valores de entalpia e entropia, ocorreu um aumento dos valores da energia livre de Gibbs com o aumento da temperatura do ar de secagem (Tabela 4). A energia livre de Gibbs é atribuída ao trabalho necessário para tornar os locais de sorção disponíveis (Nkolo Meze'e et al., 2008). Os valores positivos da energia livre de Gibbs indicam que a secagem das folhas de timbó não foi um processo espontâneo; esses valores positivos são característicos de uma reação endergônica, isto é, requerem adição de energia proveniente do meio em que se encontra o produto para que haja a reação (Corrêa et al., 2010).

\section{Conclusões}

1. O modelo matemático de secagem proposto por Midilli é, dentre aqueles testados, o que apresenta melhor ajuste sendo o selecionado para a representação da cinética de secagem das folhas de timbó.

2. O aumento da temperatura promove uma taxa maior de remoção de água nas folhas de timbó, durante a secagem.

3. O coeficiente de difusão efetivo aumenta com a elevação da temperatura do ar de secagem; esta relação pode ser descrita pela equação de Arrhenius que apresenta uma energia de ativação para a difusão líquida durante a secagem, de $81,39 \mathrm{~kJ} \mathrm{~mol}^{-1}$ para as folhas de timbó.

4. A energia livre de Gibbs aumenta com o incremento da temperatura enquanto a entalpia e a entropia decrescem.

\section{Literatura Citada}

ASABE - American Society of Agricultural and Biological Engineers. Moisture Measurement - Forages: Standard S358.2 DEC1988, R2008. In: American Society of Agricultural and Biological Engineers (ed.). Standards, Engineering Practices, and Data. St. Joseph: ASABE, 2010. p.684-685.

Babalis, S. J.; Belessiotis, V. G. Influence of the drying conditions on the drying constants and moisture diffusivity during the thin-layer drying of figs. Journal of Food Engineering, v.65, p.449-458, 2004. http://dx.doi.org/10.1016/j.jfoodeng.2004.02.005

Bourdy, G.; Michel, L. R. C. de; Roca-Coulthard, A. Pharmacopeia in a shamanistic society: the Izoceño-Guaraní (Bolivian Chaco). Journal of Ethnopharmacology, v.91, p.189-208, 2004. http://dx.doi. $\operatorname{org} / 10.1016 /$ j.jep.2003.09.013

Carlesso, V. de O.; Berbert, P. A.; Silva, R. F. da; Vianna, A. P.; Detmann, E.; Dionello, R. G. Secagem de sementes de maracujá em camada delgada. Revista Brasileira de Fruticultura, v.27, p.444-448, 2005. http://dx.doi.org/10.1590/S0100-29452005000300025

Corrêa, P. C.; Oliveira, G. H. H.; Botelho, F. M.; Goneli, A. L. D.; Carvalho, F. M. Modelagem matemática e determinação das propriedades termodinâmicas do café (Coffea arabica L.) durante o processo de secagem. Revista Ceres, v.57, p.595-601, 2010. http:// dx.doi.org/10.1590/S0034-737X2010000500005 
Corrêa, P. C.; Resende, O.; Martinazzo, A. P.; Goneli A. L. D.; Botelho, F. M. Modelagem matemática para a descrição do processo de secagem do feijão (Phaseolus vulgaris L.) em camadas delgadas. Engenharia Agrícola, v.27, p.501-510, 2007. http://dx.doi. org/10.1590/S0100-69162007000300020

Dalpasquale, V. A.; Sperandio, D. Modelo de simulação de secagem de produtos agrícolas usando entalpia do ar constante. Engenharia Agrícola, v.30, p.726-731, 2010. http://dx.doi.org/10.1590/S010069162010000400016

Goneli, A. L. D. Variação das propriedades físico-mecânicas e da qualidade da mamona (Ricinus communis L.) durante a secagem e o armazenamento. Viçosa: UFV, 2008. 186p. Tese Doutorado

Goneli, A. L. D.; Corrêa, P. C.; Oliveira, G. H. H.; Botelho, F. M. Water desorption and thermodynamic properties of okra seeds. Transactions of the ASAE, v.53, p.191-197, 2010. http://dx.doi. org/10.13031/2013.29486

Goneli, A. L. D.; Nasu, A. K.; Gancedo, R.; Araújo, W. D.; Sarath, K. L. L. Cinética de secagem de folhas de erva baleeira (Cordia verbenacea DC.). Revista Brasileira de Plantas Medicinais, v.16, p.434-443, 2014a. http://dx.doi.org/10.1590/1983-084X/13_041

Goneli, A. L. D.; Vieira, M. do C.; Vilhasanti, H. da C. B.; Gonçalves, A. A. Modelagem matemática e difusividade efetiva de folhas de aroeira durante a secagem. Pesquisa Agropecuária Tropical, v.44, p.56-64, 2014b. http://dx.doi.org/10.1590/S1983-40632014000100005

Guarim Neto, G.; Santana, S. R. A família Sapindaceae para a flora do estado de Mato Grosso do Sul, Brasil. In: Simpósio sobre Recursos Naturais e Sócio-Econômicos do Pantanal: Os Desafios do Novo Milênio, 3, 2000, Corumbá. Anais...Corumbá: Embrapa Pantanal, 2000. p.1-46.

Jideani, V. A.; Mpotokwana, S. M. Modeling of water absorption of botswana bambara varieties using Peleg's equation. Journal of Food Engineering, v.92, p.182-188. 2009. http://dx.doi.org/10.1016/j. jfoodeng.2008.10.040

Kashaninejad, M.; Mortazavi, A.; Safekordi, A.; Tabil, L. G. Thin-layer drying characteristics and modeling of pistachio nuts. Journal of Food Engineering, v.78, p.98-108, 2007. http://dx.doi.org/10.1016/j. jfoodeng.2005.09.007

Lorenzi, H.; Matos, F. J. A. Plantas medicinais no Brasil: Nativas e exóticas. 2 ed. Nova Odessa: Instituto Plantarum de Estudos da Flora Ltda., 2008. 544p.

Madamba, P. S.; Driscoll, R. H.; Buckle, K. A. The Thin-layer Drying Characteristics of Garlic Slices. Journal of Food Engineering, v.29, p.75-97, 1996. http://dx.doi.org/10.1016/0260-8774(95)00062-3
Martinazzo, A. P. Secagem, armazenamento e qualidade de folhas de Cymbopogon citratus (D. C.) Stapf. Viçosa: UFV, 2006. 140p. Tese Doutorado

Martinazzo, A. P.; Corrêa, P. C.; Resende, O.; Melo, E. de C. Análise e descrição matemática da cinética de secagem de folhas de capim-limão. Revista Brasileira de Engenharia Agrícola e Ambiental, v.11, p.301-306, 2007. http://dx.doi.org/10.1590/S1415-43662007000300009

Midilli, A.; Kucuk, H.; Yapar, Z. A new model for single-layer drying. Drying Technology, v.20, p.1503-1513, 2002. http://dx.doi. org/10.1081/DRT-120005864

Mohapatra, D.; Rao, P. S. A thin layer drying model of parboiled wheat. Journal of Food Engineering, v.66, p.513-518, 2005. http://dx.doi. org/10.1016/j.jfoodeng.2004.04.023

Moreira, R.; Chenlo, F.; Torres, M. D.; Vallejo, N. Thermodynamic analysis of experimental sorption isotherms of loquat and quince fruits. Journal of Food Engineering, v.88, p.514-521, 2008. http:// dx.doi.org/10.1016/j.jfoodeng.2008.03.011

Nkolo Meze'e, Y. N.; Noah Ngamveng, J.; Bardet, S. Effect of enthalpyentropy compensation during sorption of water vapour in tropical woods: The case of bubinga (Guibourtia tessmanii J. L'Eonard; G. Pellegriniana J. L.). Thermochimica Acta, v.468, p.1-5, 2008. http:// dx.doi.org/10.1016/j.tca.2007.11.002

Panchariya, P. C.; Popovic, D.; Sharma, A. L. Thin-layer modeling of black tea drying process. Journal of Food Engineering, v.52, p.349357, 2002. http://dx.doi.org/10.1016/S0260-8774(01)00126-1

Prates, M. F. O.; Reis, R. C. dos; Devilla, I. A.; Faria, R. Q.; Lima Junior, A. F. Cinética de secagem de folhas de Solanum lycocarpum A. St.-Hil. (fruta-de-lobo). Revista Brasileira de Plantas Medicinais, v.14, p.514521, 2012. http://dx.doi.org/10.1590/S1516-05722012000300014

Radünz, L. L.; Amaral, A. S. do; Mossi, A. J.; Melo, E. de C.; Rocha, R. P. Avaliação da cinética de secagem de carqueja. Engenharia na Agricultura, v.19, p.19-27, 2011.

Rizvi, S. S. H. Thermodynamic properties of foods in dehydration. In: Rao, M. A.; Rizvi, S. S. H. Engineering properties of foods. New York: Academic Press, 1995. p.223-309.

Siqueira, V. C.; Resende, O.; Chaves, T. H. Drying kinetics of Jatropha seeds. Revista Ceres, v.59, p.171-177, 2012. http://dx.doi. org/10.1590/S0034-737X2012000200004

Tabaldi, L. A.; Vieira, M. C.; Zárate, N. A. H.; Silva, L. R.; Gonçalves, W. L. F.; Pilecco, M.; Formagio, A. S. N.; Gassi, R. P.; Padovan, M. P. Cover crops and their effects on the biomass yield of Serjania marginata plants. Ciência Rural, v.42, p.614-620, 2012.

Zogzas, N. P.; Maroulis, Z. B.; Marinos-Kouris, D. Moisture diffusivity data compilation in foodstuffs. Drying Technology, v.14, p.22252253, 1996. http://dx.doi.org/10.1080/07373939608917205 